\title{
Tales of experimentation
}

\section{Rom Harré: Pavlov's dogs and Schrödinger's cat: Scenes from the living laboratory. Oxford: Oxford University Press, 2009, xiii+322 pp, £16.99 HB}

\section{Mieke Boon}

Published online: 21 September 2010

(C) The Author(s) 2010. This article is published with open access at Springerlink.com

Usually, we think of scientific laboratories as technological environments consisting of mainly inorganic materials such as glass or metal containers, wires, electronic apparatus, technical tools, and diverse chemicals, but in many cases also living or dead organisms are used for which no technological variants exist. Harré's general aim of studying 'scenes from the living laboratory' is to show the many different ways in which living materials have been used as tools for experimenting. The book presents a broad palette of colorful stories. In all these stories, organic materials, whether alive or dead, were used as instruments for performing measurements, as apparatus with which experiments were carried out, or as sources for recovering specific ingredients used as indicators in scientific or industrial applications, and sometimes as objects of study for their own sake.

Harré distinguishes between instruments, which are all sorts of detectors and measuring devices, and apparatus, which are devices that isolate parts of the world to be studied. Instruments are causally related to the rest of the world because the interaction between instruments and world gives quantitative or qualitative information about the latter. Chapters 2 and 3 present the examples of instruments, while Chapters 4 through 8 deal with apparatus. Apparatus help scientists to isolate aspects of a complex reality. In addition, they create working models of natural processes. According to Harré, apparatus are conceptually related to the world because, by drawing analogies, we reason from what happens in the laboratory to how things might be in nature.

Chapters 2 and 3 of the book describe several cases in which animals and plants are used in detecting and measuring. One such case is the use of the hind legs of frogs as the essential core of experimental devices in studies of electrical phenomena by Luigi Galvani, and later, Alessandro Volta. This case also illustrates

\footnotetext{
M. Boon $(\bowtie)$

Department of Philosophy, University of Twente, P.O. Box 217, 7500 AE Enschede,

The Netherlands

e-mail: M.Boon@utwente.nl
} 
an important feature of the dynamics of experimentation in science: Initially, manipulating and understanding the phenomenon is itself the object of study (e.g., the behavior of the muscles of the frog legs in studying electrical phenomena); then, it may be used as an instrument for detection and/or measurement (e.g., the frog legs became the instruments by which electrical currents could be detected). Another example of this dynamics is the experimental use of litmus containing lichens. Initially, the object of study was to find a recipe for the extraction of litmus from lichens; later, litmus became a widely used instrument for detecting acidity and alkalinity. Other examples of organic material used in detecting are: animals that served the purpose of finding out whether humans would survive conditions in the upper atmosphere and even outer space; milkweed that was used as a detector of ozone in air; canaries used as detectors of carbon monoxide in mines; and transgenic fish that display different fluorescent colors when they encounter specific toxic substances were used as detectors of those substances. Not only toxic conditions and pollutants but also climate and change of climate can be detected by means of organic matter: the occurrence of fossils and bones of specific kinds of animals in specific areas or layers (so-called paleo-botany) is related to a specific climate and can thus be used in detecting the occurrence of climate change in the history of the earth.

Chapter 4 is about experimental studies into the nature of blood in animals and sap in plants, and the mechanisms of the blood and sap stream, as well as of how to intervene in them. Harré stresses that the experimental apparatus was the living body itself-more particularly in the case of blood, the bodies of domestic animals. He describes the well-known history of William Harvey's experimental demonstration of the circulation of blood, which is one of the horrifying examples of vivisection in an era where anesthesia was unknown. Harvey emphasized that his claims had been established experimentally, that is by dissection of living animals to reveal the circulatory system in action. Following up on those findings, Richard Lower, in the seventeenth century, injected colored liquids into the venous system just at the moment in which an animal was killed, which allowed the whole of the blood vascular system to be observed.

In describing these episodes, Harré points toward another crucial feature of the experimental dynamics in the 'living laboratory', namely that scientists draw on analogies of methods, techniques, and concepts from existing (physical) sciences. These analogies play a vital role in coming up with research questions, techniques, and hypotheses.

Harré's descriptions also imply another feature of the dynamics of experimenting in science, namely that, in many cases, experimentation is not directed by previously stated hypotheses or research questions. Instead, the experimental apparatus itself fuels the imagination about possibilities of manipulating it. Dogs or horses, as experimental apparatus for examining bloodstreams, bring to the mind of a researcher the possibility of injecting various substances and even the blood of other animals. In a similar, although less cruel way, due to his humane attitude and the availability of anesthesia, Ivan Petrovich Pavlov developed surgical techniques to sample physiological processes, particularly of digestion. He instigated the construction of a laboratory where surgical procedures could be carried out on 
his dogs, treating the dog as an apparatus with portals through which the secretions involved in various phases of the digestive process could be extracted.

Harré mentions that Pavlov strongly and publicly opposed the 'crude vivisection' of animals and was a foremost defender of their humane use in scientific studies. Yet, the dynamics of experimental research suggests that the scientific attitude of researchers in the 'living laboratory' is often very similar to that in the 'inorganic laboratory'. Admittedly, the unscrupulous tendency to manipulate living animals has led to medical technologies such as blood transfusion, surgical techniques, and heart transplants. Nevertheless, Harré suggests-although mostly 'between the lines'-that the 'living laboratory' may be in need of a more morally conscious scientific attitude.

The next three chapters are a bit of a hotchpotch of cases because each chapter gathers stories from widely diverging fields such as physiology and the medical sciences, linguistics, psychology, biochemistry, and sometimes also from the relations between these fields (e.g., between biochemistry and psychology). Their organization in themes such as explorative experimental studies (Chapter 5), testing hypotheses (Chapter 6), and model-making (Chapter 7) is somewhat artificial since most of these aspects apply to almost all of the cases in the book. For all that, when taken by themselves, the described stories are interesting, not in the least because they cover such a wide range of scientific fields and purposes and also because Harré tells stories of which we often only know just bits and pieces. Examples are: the human ear of the Vacanti mouse; Gardner's program of animal language acquisition; the well-known Milgram experiments on humans; Pavlov's physiological surgery with dogs; Harry Harlow's maternal separation and social isolation experiments on rhesus monkeys; Christiaan Barnard's forty-eight trial heart transplants with dogs; the experimental use of mice in relating psychological features such as depression and aggression with their genetic makeup or biochemistry; Barry Marshall's use of his own body as an experimental apparatus for proving the role of a specific bacterium.

In these chapters about experimental apparatus, Harré introduces the notion of intensive design. By this notion, he aims at an account of the method of induction that would fit actual scientific practices. He rejects the traditional idea of 'induction by enumeration'. To the contrary, the stories illustrate that researchers often prove their point by a few or just one exemplar. Results established on a few examples are taken to be typical and generalized to all the members of the same species, and in some cases, even to other species including human beings. The Vacanti brothers proved their point with just one case.

Unfortunately, besides stressing the point that in many cases generalization goes by just one exemplar (i.e., by intensive design), Harré leaves implicit the specific character of intensive design-neither does he explain why scientists believe that such generalizations are justified.

The stories presented by Harré show, for instance, that research questions, experimental methods, and experimental apparatus and techniques are built on top of one another in an ongoing dynamics: questions are raised (e.g., what is the function of blood); experimental apparatus and techniques that enable intervention, observation, and measurement are developed (e.g., connecting the bloodstream of 
two dogs or injecting fluids into the bloodstream); new observations are made; resistances and problems are encountered (e.g., injection of blood in bloodstream leads to coalescence of the blood) and solved by adapting the experimental apparatus and techniques; new possibilities of intervention are suggested by the reliably functioning apparatus (e.g., blood transfusion); and new measurements or apparatus are envisioned and developed by analogy with physical experiments (e.g., the measurement of the blood pressure).

This picture of scientific experimentation goes against a traditional picture in the philosophy of science according to which (1) experiments are set up in a straightforward manner to test a hypothesis, and (2) evidence is gathered by enumerative induction. Regarding point (1), in many cases, experiments are set up by the mere possibility of intervention, and from these experiments new hypotheses arise. Moreover, experimental apparatus are adapted until the technique 'functions properly'. These adaptations are guided by expected or desired outcomes. The case of Lysenko's attempt to prove the Lamarckian hypothesis that the way of life of a plant or animal in one generation can lead to changes in the genetic material, which can then be passed onto subsequent generations (extensively described by Harré in Chapter 9), illustrates that this non-eliminable feature of experimental practices can lead to scientific fraud.

Regarding the point of why generalizations from just a few cases are justified, Harré's suggestion of a conceptual relation between the experimental apparatus that result from a process of intensive design, on the one hand, and all members of a species or even other species, on the other, is insufficient. In generalizations from the model apparatus to other target systems such as from the functioning of the heart of these few dogs to that of all dogs or even to that of all mammals including human beings or from the biochemistry of mouse to that of all mammals, scientists must assume that the physical structures are sufficiently similar, rather than only conceptually analogous as Harré suggests.

The presented studies are very much worth reading, partly because Harré compiled them into concise, attractive, and sufficiently detailed stories, and also because this compilation goes beyond the canonical cases used in the philosophy of science literature, thus illustrating the enormous variety of experimental approaches in science. Describing these extra-canonical stories with a focus on how and why scientists design tools for experimenting brings to the surface new philosophical issues and puts into question some well-accepted traditional ideas.

A limitation of this book is that Harré does not explicitly address obvious philosophical questions of the 'living laboratory', such as whether the use of organic, living or dead, materials makes laboratory research fundamentally different or whether it raises new philosophical issues with regard to, e.g., scientific methodology and the justification of knowledge, the use of instruments and experimental models, and the relation between scientific research and technological applications. These are the kinds of questions that come to mind when reading Harré's book from a philosophy of science perspective. The stories suggest that, since the experimental dynamics are very similar, there is no fundamental difference between the living and the 'inorganic laboratory'. 
Yet, focus on the 'living laboratory' leads to epistemological issues that are much more pressing due to their entanglement with moral issues concerning the use of organic beings in scientific experiments. In the last chapter of this book, we learn that these moral issues are among Harré's concerns. He takes it that reflecting on them requires detailed and rich descriptions on a case-by-case basis, not only of the experimental setup but also of the researchers and their purposes. Indeed, the stories present a wealth of possibilities for raising and analyzing these issues, but Harré usually does not address them in much depth and in many cases leaves them 'hanging in the air'. Nevertheless, the content of the book enriches our imagination and moral intuitions and shows a potential of much further work in the philosophy of science, in particular by presenting a rich source of case descriptions in which epistemological and moral issues are entangled and call out for further analysis.

Open Access This article is distributed under the terms of the Creative Commons Attribution Noncommercial License which permits any noncommercial use, distribution, and reproduction in any medium, provided the original author(s) and source are credited. 